\title{
The relevance of maternal cardiovascular situation before labor in the outcome of birth: an observational study.
}

\author{
Daniele Farsetti ${ }^{1}$, Grazia Tiralongo ${ }^{2}$, Ilaria Pisani ${ }^{2}$, Damiano Lo Presti ${ }^{2}$, Giulia Gagliardi ${ }^{3}$, \\ Barbara Vasapollo ${ }^{2}$, Gian Paolo Novelli ${ }^{4}$, and Herbert Valensise ${ }^{1}$ \\ ${ }^{1}$ University of Rome Tor Vergata \\ ${ }^{2}$ Policlinico Casilino \\ ${ }^{3}$ Casilino General Hospital \\ ${ }^{4}$ Fondazione PTV Policlinico Tor Vergata
}

October 1, 2020

\begin{abstract}
Objective: To assess if maternal hemodynamics assessment in healthy women, at the end of pregnancy, before the labor onset, could predict the development of complications during labor. Design: Prospective observational study. Settings: Department of Obstetrics and Gynecology of Casilino Hospital in Rome, University of Tor Vergata. (September 2016 - December 2017) Population: 395 healthy women not in labor at term of pregnancy. Methods: Univariate and multivariate binary logistic regression analysis of association between hemodynamic variables and delivery complications, adjusted for significant prenatal variables. Main Outcome Measures: ORs for hemodynamic parameters and identification of independent risk factors in the prediction of adverse outcome. Results: we observed adverse outcomes in 45 patients (11.39\%). Women who developed maternal or fetal complications during delivery were mainly nulliparous $(93.33 \%$ vs $72.29 \%, \mathrm{p}<0.01)$, showed higher values of systemic vascular resistances (SVR) (1368.32 \pm 228.50 . vs $1260.34 \pm 271.94$ d.s.cm-5, p=0.01) and lower values of cardiac output (CO) $(5.38 \pm 0.77$ vs $5.80 \pm 1.20 \mathrm{~L} / \mathrm{min}, \mathrm{p}=0.02)$, compared with women who did not develop complications. ROC curve analysis identified the best cut-offs to predict complicated delivery: SVR > 1135 d.s.cm-5 (OR 7.87, CI 95\% 2.39-25.92, p<0.01), CO [?]5.6 L/min (OR 2.38; CI 95\% 1.21-4.68, p<0.01), diastolic blood pressure $>79 \mathrm{mmHg}(\mathrm{OR} 1.89$, CI 95\%.1.01-3.54, $\mathrm{p}=0.04)$, Potential-Kinetic energy Ratio $>22$ (OR 2.32, 0.95-5.64, $\mathrm{p}=0.04)$. The multivariate logistic regression analysis identified as independent predictors of complication the SVR, Flow Time corrected and parity. Conclusion: the assessment of maternal hemodynamics at term of pregnancy could increase the capacity to predict patients at risk during labor.
\end{abstract}

THE RELEVANCE OF MATERNAL CARDIOVASCULAR SITUATION BEFORE LABOR IN THE OUTCOME OF BIRTH: AN OBSERVATIONAL STUDY.

Daniele Farsetti ${ }^{1,2}$

Grazia Maria Tiralongo ${ }^{2}$

Ilaria Pisani ${ }^{2}$

Damiano Lo Presti ${ }^{2}$

Giulia Gagliardi ${ }^{2}$

Barbara Vasapollo ${ }^{2}$

Gian Paolo Novelli ${ }^{3}$

Herbert Valensise ${ }^{1,2}$ 
1. University of Rome Tor Vergata, Surgical Sciences, Rome, Italy

2. Casilino General Hospital, Obstetrics and Gynecology, Rome, Italy

3. Fondazione PTV Policlinico Tor Vergata, Cardiology, Rome, Italy

Corresponding author:

Daniele Farsetti

dan.farsetti@gmail.com

+393395238927

Running Title : Maternal Hemodynamics in Outcome of Birth

Objective : To assess if maternal hemodynamics assessment in healthy women, at the end of pregnancy, before the labor onset, could predict the development of complications during labor.

Design : Prospective observational study.

Settings : Department of Obstetrics and Gynecology of Casilino Hospital in Rome, University of Tor Vergata. (September 2016 - December 2017)

Population : 395 healthy women not in labor at term of pregnancy.

Methods : Univariate and multivariate binary logistic regression analysis of association between hemodynamic variables and delivery complications, adjusted for significant prenatal variables.

Main Outcome Measures: ORs for hemodynamic parameters and identification of independent risk factors in the prediction of adverse outcome.

Results : we observed adverse outcomes in 45 patients (11.39\%). Women who developed maternal or fetal complications during delivery were mainly nulliparous ( $93.33 \%$ vs $72.29 \%, \mathrm{p}<0.01$ ), showed higher values of systemic vascular resistances (SVR) $(1368.32 \pm 228.50$. vs $1260.34 \pm 271.94$ d.s.cm- $5, \mathrm{p}=0.01)$ and lower values of cardiac output $(\mathrm{CO})(5.38 \pm 0.77$ vs $5.80 \pm 1.20 \mathrm{~L} / \mathrm{min}, \mathrm{p}=0.02)$, compared with women who did not develop complications. ROC curve analysis identified the best cut-offs to predict complicated delivery: SVR $>1135$ d.s.cm-5 (OR 7.87, CI 95\% 2.39-25.92, p<0.01), CO [?]5.6 L/min (OR 2.38; CI 95\% 1.21-4.68, p<0.01), diastolic blood pressure $>79 \mathrm{mmHg}(\mathrm{OR} 1.89$, CI 95\% 1.01-3.54, $\mathrm{p}=0.04)$, Potential-Kinetic energy Ratio $>22$ (OR 2.32, 0.95-5.64, $\mathrm{p}=0.04$ ). The multivariate logistic regression analysis identified as independent predictors of complication the SVR, Flow Time corrected and parity.

Conclusion : the assessment of maternal hemodynamics at term of pregnancy could increase the capacity to predict patients at risk during labor.

Key words: Labour: management, obstetric haemorrhage, maternal physiology, fetal medicine: uteroplacental insufficiency, doppler ultrasound.

Funding statement : no funding.

TWEETABLE ABSTRACT

The assessment of maternal hemodynamics at the end of pregnancy could predict complications during labor.

\section{INTRODUCTION}

The development of an accurate diagnostic test to identify the risk of an adverse outcome during labor has long been the greatest challenge for obstetricians. The modern risk assessment of developing complications during the delivery is based on maternal age, socio-economic conditions, ethnicity, educational level and obstetrical history11Essex HN, Green J, Baston H, Pickett KE. Which women are at an increased risk of a caesarean section or an instrumental vaginal birth in the UK: an exploration within the Millennium Cohort Study. BJOG. 2013 May;120(6):732-42.'22Lean SC, Derricott H, Jones RL, Heazell AEP. Advanced 
maternal age and adverse pregnancy outcomes: A systematic review and meta-analysis.. PLoS One. 2017 Oct 17;12(10):e0186287..

The recent knowledge that some term fetuses, with estimated weight appropriate for gestational age, can suffer of placental dysfunction, increased the use of cerebroplacental ratio (CPR) to identify fetuses with a higher risk of adverse pregnancy outcome33Prior T, Mullins E, Bennett P, Kumar S. Prediction of intrapartum fetal compromise using the cerebroumbilical ratio: a prospective observational study. Am J Obstet Gynecol. 2013 Feb;208(2):124.e1-6,44Morales-Rosello J, Khalil A, Morlando M, Bhide A, Papageorghiou A, Thilaganathan B. Poor neonatal acid-base status in term fetuses with low cerebroplacental ratio. Ultrasound Obstet Gynecol. 2015 Feb;45(2):156-61.. Nevertheless, according to the modern review studies, the role of the CPR in the prediction of perinatal outcome is not clear55Conde-agudelo A, Villar J, Kennedy SH, Papageorghiou AT. Predictive accuracy of cerebroplacental ratio for adverse perinatal and neurodevelopmental outcomes in suspected fetal growth restriction: systematic review and meta-analysis. Ultrasound Obstet Gynecol 2018; 52: 430-441..

The maternal cardiovascular function has shown to play a key role during all the course of pregnancy. An impaired cardiovascular adaptation could compromise in a number of ways the pregnancy: increasing the risk of hypertensive disorder66Pisani I, Tiralongo GM, Lo Presti D, Gagliardi G, Farsetti D, Vasapollo B et al. Correlation between maternal body composition and haemodynamic changes in pregnancy: different profiles for different hypertensive disorders. Pregnancy Hypertens. 2017 Oct;10:131-134. 77 Gagliardi G, Tiralongo GM, Lo Presti L, Pisani I, Farsetti D, Vasapollo et al. Screening for pre-eclampsia in the first trimester: role of maternal hemodynamics and bioimpedance in non-obese patients. Ultrasound Obstet Gynecol 2017; 50: 584-588, fetal growth restriction88Tiralongo GM, Pisani I, Vasapollo B, Khalil A, Thilaganathan B, Valensise H. Effect of a nitric oxide donor on maternal hemodynamics in fetal growth restriction. Ultrasound Obstet Gynecol. 2018 Apr;51(4):514-518., or even preterm delivery99Valensise H, Farsetti D, Lo Presti D, Pisani I, Tiralongo GM, Gagliardi G et al. Preterm delivery and elevated maternal total vascular resistance: signs of suboptimal cardiovascular adaptation to pregnancy? Ultrasound Obstet Gynecol. 2016 Oct;48(4):491-495..

In a previous pioneer study led by our group, we observed how the assessment of maternal cardiovascular adaptation to labor might identify patients at risk of developing complications1010Valensise $\mathrm{H}$, Tiralongo GM, Pisani I, Farsetti D, Lo Presti D, Gagliardi G et al. Maternal cardiac output in early labour: a possible link with obstetrics risks? Ultrasound Obstet Gynecol. 2017 Feb 25.. In particular, women with low cardiac output (CO) and increased systemic vascular resistance (SVR) in the early stage of labor had 8-10-fold increased risk of developing intrapartum complications. Our hypothesis was that a reduced uterine perfusion could cause consequent fetal sufferance.

The possibility to identify the prenatal risk factors in a term pregnancy, especially before the begin of labor, could be a great help in the management. The aim of this study is to verify if healthy women with increased SVR at the end of pregnancy, before the labor onset, show an augmented risk to develop complications during labor. Secondly, we want to identify the best independent predictors of adverse outcome.

\section{METHODS}

This prospective study was conducted in the Department of Obstetrics and Gynecology of Casilino Hospital in Rome, University of Tor Vergata. During the period between September 2016 and December 2017, we enrolled 395 patients not in labor.

We used strict inclusion and exclusion criteria. The inclusion criteria were:

- singleton pregnancy,

- certain dating of pregnancy by I trimester ultrasound using crown-rump length (CRL),

- gestational age $>37+0$,

- estimated fetal birth weight $>10$ th centile,

- absence of fetal and maternal disease at enrollment.

Pregnant women were excluded if a maternal or fetal disease was present (diabetes, hypertensive disorders, 
IUGR, fetal malformation, etc.), if the pregnancy was the result of assisted reproduction therapy or if an elective C-section due to maternal request or breech presentation was scheduled.

Once the consensus was obtained, the anamnestic, anthropometric and demographic information were gathered. For each patient, the following parameters were collected: maternal age, height, weight, BMI, last menstrual period, gestational age corrected by first trimester ultrasound, obstetrical history and smoking.

An ultrasound exam was performed to measure the Pulsatility Index of Umbilical Artery, the Pulsatility Index of Middle Cerebral Artery and the Amniotic Fluid Index. Ultrasound examination was performed with a $3.5-\mathrm{MHz}$ sector ultrasound transducer with the high-pass filter set at $100 \mathrm{~Hz}$, in a moment of absence of fetal breathing and body movements. The umbilical artery, middle cerebral artery and uterine arteries were studied using colour Doppler according to the most modern standard protocol11Bhide A, Acharya G, Bilardo CM, Brezinka C, Cafici D, Hernandez-Andrade E et al. ISUOG practice guidelines: use of Doppler ultrasonography in obstetrics. Ultrasound Obstet Gynecol. 2013 Feb;41(2):233-39..

The CPR was calculated by dividing the Doppler indices of the middle cerebral artery (MCA) by the umbilical artery (UA). The UA and MCA pulsatility index (PI) was calculated according to a standard protocol22Bahlmann F, Reinhard I, Krummenauer F, Neubert S, Macchiella D, Wellek S. Blood flow velocity waveforms of the fetal middle cerebral artery in a normal population: reference values from 18 weeks to 42 weeks of gestation. J Perinat Med 2002; 30: 490 - 501..

The maternal haemodynamics was assessed by UltraSonic Cardiac Output Monitor (USCOM(r), USCOM Ltd., Coffs Harbour, Australia). It is a non-invasive method that uses a continuous-wave Doppler to determine CO. It has demonstrated a comparable accuracy to invasive methods (Swan-Ganz catheter) and the USCOM measurements are well correlated with echocardiographic assessments33Bijl RC, Valensise H, Novelli GP, Vasapollo B, Wilkinson I, Thilaganathan B et al; International Working Group on Maternal Hemodynamics. Methods and considerations concerning cardiac output measurement in pregnant women: recommendations of the International Working Group on Maternal Hemodynamics. Ultrasound Obstet Gynecol. 2019 Jul;54(1):35-50.44Kager CC, Dekker GA, Stam MC. Measurement of cardiac output in normal pregnancy by a non- invasive two-dimensional independent Doppler device. Aust N Z J Obstet Gynaecol. 2009 Apr;49(2):142-4.55Namara H, Barclay P, Sharma V. Accuracy and precision of the ultrasound cardiac output monitor (USCOM 1A) in pregnancy: comparison with three-dimensional transthoracic echocardiography. Br. J. Anaesth. (2014) 113 (4): 669-676.. The measurements were performed by two trained operators, in the same room, under standardised conditions, with a patient in a semi-recumbent position, after she had rest in this position at least 15 minutes. Blood pressure was obtained before the hemodynamic measurement, using an automatic blood pressure monitor with the patient in a semi-recumbent position and using an appropriately sized cuff.

All the examinations were made from the suprasternal notch targeting the aortic valve using a $2.2 \mathrm{MHz}$ Transducer, with patients in left lateral recumbent position.

We collected the following USCOM parameters: systolic blood pressure (SBP), diastolic blood pressure (DBP), systemic vascular resistance (SVR), systemic vascular resistance index (SVRI), cardiac output (CO), cardiac index (CI), stroke volume (SV), Smith-Madigan inotropy index (INO), flow time corrected (FTc), potential to kinetic energy ratio (PKR).

At the day of childbirth, we collected information about the labor and the delivery in order to identify any maternal or neonatal complications: gestational age at time of delivery, request of epidural analgesia, labor onset type (spontaneous or with induction), childbirth type (spontaneous, operative vaginal delivery, csection) and any obstetrical indications, type of placental delivery, loss of blood and need of blood transfusion, neonatal sex, neonatal weight, 1-min Apgar and 5-min Apgar.

The induction of labor, if was required by internal routine antenatal management protocols, was performed with dinoprostone $10 \mathrm{mg}$ vaginal insert and, after 24 hours, if the labor failed to start the vaginal insert was removed and after 30 minutes the oxytocin infusion started according to "low-dose" protocol66Fondazione 
Confalonieri Ragonese su mandato di SIGO, AOGOI, AGU. Induzione al travaglio di parto. 2016.

A delivery was considered "complicated" if just one of these conditions was present:

- caesarean section or vaginal operative delivery for pathological CTG77National Institute for Health and Care Excellence. Interpretation of cardiotocograph traces, NICE guideline 2017.,

- major post-partum haemorrhage (PPH) with uterine atony,

- 5- min Apgar score < 7,

- NICU admission.

The PPH is defined as the loss of $500 \mathrm{ml}$ or more of blood from the genital tract within 24 hours of the birth of a baby88Mousa HA, Blum J, Abou El Senoun G et al. Treatment for primary postpartum haemorrage Cochrane Database Syst Rev2014;(2)CD003249 Mousa HA, Blum J, Abou El Senoun G, Shakur H, Alfirevic Z. Treatment for primary postpartum haemorrhage. Cochrane Database Syst Rev 2014;(2):CD003249. Mousa HA, Blum J, Abou El Senoun G, Shakur H, Alfirevic Z. Treatment for primary postpartum haemorrhage. Cochrane Database Syst Rev 2014;(2):CD003249. The uterine atony is defined as the condition when the uterus fails to contract after the delivery of the baby99Breathnach F, Geary M. Uterine atony: definition, prevention, nonsurgical management, and uterine tamponade. Semin Perinatol. 2009 Apr;33(2):82-7.

\section{STATISTICAL ANALYSIS}

Clinical continuous data were expressed as mean and standard deviation, categorical variables were expressed as number and percentage.

Comparison continuous variables were performed with Student's t-test for unpaired data. For the categorical variables we performed a Chi-Squared test for the comparison of two proportions (from independent samples).

We performed a logistic univariate regression analysis to assess the association of complications during the delivery and cardiovascular or maternal demographic parameters.

A receiver operating characteristics (ROC) curve was constructed for each hemodynamic parameter, to identify the best cut-off and convert the continuous variables into categorical. We performed a univariate binary logistic regression analysis and odds ratios were calculated.

In conclusion, a multivariate binary logistic regression analysis was performed including the hemodynamic and maternal significant variables. Since CO and blood pressure values were included in the SVR formula, and are related to each other, only SVR was included in the multivariate binary logistic regression analysis.

The statistical analysis was performed using MedCalc Statistical Software (MedCalc Software bvba, Ostend, Belgium; https://www.medcalc.org; 2016). Statistical significance was set to $\mathrm{p}<0,05$.

\section{RESULTS}

We observed adverse outcomes in 44 patients (11.39\%). In the table 1 are summarized the maternal characteristics at the time of enrollment.

There were no differences at the time of the enrollment between women who will experience complications and patients who won't in terms of gestational age, maternal age, BMI, percentage of smokers and CerebroPlacental Ratio (CPR) centile. Nevertheless, the rate of multiparity is higher in women who delivered without complications $(27.71 \%$ vs $6.67 \%, \mathrm{p}<0.01)$.

Women who will complicate during the labor had significantly higher DBP $(77.36+-9.51 \mathrm{mmHg}$ vs $74.14+-$ $9.21 \mathrm{mmHg}, \mathrm{P}=0.03)$, higher SVR (1368.32+-228.50 d.s.cm-5 vs $1260.34+-271.94$ d.s.cm-5, $\mathrm{p}=0.01)$, higher SVRI (2544.42+-518.23 d.s.cm ${ }^{-5} . \mathrm{m}^{2}$ vs $2378.23+-530.54$ d.s. $\left.\mathrm{cm}^{-5} . \mathrm{m}^{2}, \mathrm{p}=0.05\right)$ and lower CO (5.38+$0.77 \mathrm{l} / \mathrm{min}$ vs $5.80+-1.20 \mathrm{l} / \mathrm{min}, \mathrm{p}=0.02)$ compared to controls. No significant differences were observed between the two groups in terms of SBP, SV, CI, INO, FTc, PKR (Table 1).

The group of women with complicated delivery had lower rate of induction ( $8.89 \%$ vs $24 \%, \mathrm{p}<0.01)$, increased percentage of urgent C-section ( $68.89 \%$ vs $12.57 \%, \mathrm{p}<0.01)$ and operative delivery $(20.00 \%$ vs $8.29 \%, \mathrm{p}<0.01)$, 
lower rate of clear amniotic fluid (73.33\% vs 90.57\%) and lower values of Apgar 1-min (8.00+-1.51 vs 8.69+$0.69, \mathrm{p}<0.01)$ and Apgar 5-min $(9.44+-0.73$ vs $9.72+-0.75, \mathrm{p}<0.02)$, compared to controls.

There were no differences in terms of gestational age at the time of delivery and the rate of epidural analgesia $(71.11 \%$ vs $66.57 \%)$.

Table 2 and 3 reports the univariate logistic regression analysis for each continuous and categorical variable, including hemodynamic categorical variable obtained after identification of the cut-off through the ROCcurve analysis.

We perform a multivariate logistic regression analysis with multiparity and significant hemodynamic variables and we adjusted the cardiovascular effects for the maternal height that showed a significant association with outcome. The ORs obtained from the multivariate logistic regression analysis are summarized in the table 4. Independent predictors for delivery complications were parity, SVR and FTc.

\section{DISCUSSION}

\section{Main Findings}

In this study we highlight the importance of pre-labor hemodynamic assessment in the identification of patients at risk of delivery complications. In particular, our data showed that SVR $>1135$ d.s.cm- 5 and FTc $>365 \mathrm{~ms}$ are independent predictors of adverse outcome during labor.

\section{Strengths}

One strength of this study is that the hemodynamic assessment was performed in standardized situation, without confounding factors (labor pain, different room conditions, etc.) that could compromise hemodynamic parameters. Unlike to our previous study, evaluating maternal hemodynamics before the labor onset, has been possible to standardize the evaluation in order to assess if the hemodynamic profile, at rest, in a non-stress condition, is different in women at risk of delivery complications.

Another key strength is that the labor management team was not informed of hemodynamic evaluation results collected before the labor onset.

\section{Limitations}

This study was limited by the absence of blood gas analysis of the newborn that could increase our ability to identify fetal hypoxia.

We intentionally excluded the intrapartum variables from the multivariate logistic regression analysis, because the aim of this study was to identify antenatal risk factor for development of complications during labor.

\section{Interpretation}

The results of this study are in line with most relevant scientific findings on this subject. We previously demonstrated a greater incidence of complications in women with values of SVR higher than 1069 d.s.cm- 5 in the early stage of labor ${ }^{10}$. Kalafat e al. demonstrated that higher SVR correlates with an increased risk of operative delivery due to presumed fetal compromise in women undergoing induction of labor for several indications11Kalafat E, Barratt I, Nawaz A, Thilaganathan B, Khalil A. Maternal cardiovascular function and risk of intrapartum fetal compromise in women undergoing induction of labor: a pilot study. Ultrasound Obstet Gynecol. 2019 Nov 11.. Even in our study the SVR value is the best independent risk predictor for delivery complications and shows a strong association when is higher than 1135 d.s.cm ${ }^{-5}$ (OR = 9.01).

In our study, women who developed complications during the labor, showed increased SVR, reduced CO and higher PKR. The FTc, which represents the time of the left ventricular systole and is correlated to preload and cardiac performance, is apparently an independent predictor of complications on multivariate analysis when is higher than $365 \mathrm{~ms}$. This hemodynamic pattern, characterized by a high resistance and hypodynamic 
circulation, is characteristic of a cardiovascular maladaptation and this condition is significantly correlated with the development of fetal sufferance during the labor.

We can only hypothesize which is the etiopathological mechanism that determines the development of complications during the labor in patients with suboptimal cardiovascular status even before labor starts. The cardiovascular adaptation to the pregnancy is fundamental for adequate perfusion of the uteroplacental unit during the pregnancy and prevent placental syndromes22Valensise H, Novelli GP, Farsetti D, Vasapollo B. Cardiac function. In: Lees C, Gyselaers W, editors. Maternal hemodynamics. Cambridge (UK): Cambridge University Press; 2018.

In normal pregnancies the vascular remodelling leads to a SVR reduction and a CO increment that are fundamental for a correct uterine perfusion and to prevent fetal hypoxia. During the labor there is an increase in demands on the cardiovascular system, in particular CO increases by $30 \%$ in the first stage of labor and by over $50 \%$ in the second stage ${ }^{21}$. Women with high SVR and poor cardiac performance at term have not fit properly to the pregnancy and have an increased risk to develop intrapartum complications. The poor cardiac performance could be the cause of a reduced uteroplacental perfusion and a consequence fetal sufferance. The subclinical alterations in cardiac function, in apparently healthy women, could compromise the delivery outcome.

Even in our results, the multiparity is a protective factor for the development of maternal and fetal adverse outcome. The OR value is superimposable to the OR calculated by Kalafat et $\mathrm{al}^{20}$. The maternal hemodynamic parameters, adjusted for multiparity and maternal height, showed to be independent risk factors for adverse outcome.

The CPR has been demonstrated to be able to identify fetal at risk for distress during labor, even in AGA fetuses $33^{22}$ DeVore GR. The importance of the cerebroplacental ratio in the evaluation of fetal well-being in SGA and AGA fetuses. Am J Obstet Gynecol 2015; 213: 5-15. Nevertheless, in our results the CPR value, adjusted for the gestational age, didn't show significant difference in patients who will develop complications.

\section{CONCLUSION}

In conclusion, women with high resistance and hypodynamic circulation (characterized by reduced $\mathrm{CO}$ and increased SVR, PKR and FTc) at the end of pregnancy, before the onset of labor, appear to be at much greater risk of developing delivery complications. For this reason, the maternal hemodynamic assessment at the end of pregnancy could improve the ability to predict adverse outcome during labor in low-risk women. These patients, which wouldn't have been identify with traditional method, could benefit from a closer surveillance to prevent maternal and fetal complications.

\section{DISCLOSURE OF INTERESTS}

No potential conflict of interest was reported by the authors.

\section{CONTRIBUTION TO AUTHORSHIP}

DF contributed to counselling, data collection, statistical analysis and to writing the article. HV contributed to the conception and design of the study. GPN contributed to the statistical analysis. GMT, IP, DLP, GG and $\mathrm{BV}$ contributed to interpretation of the analysis and data collection.

\section{DETAILS OF ETHICS APPROVAL}

For this observational study IRB approval was obtained preliminarily. Informed consent was obtained from each woman before enrollment. Ethical approval was obtained from the Research Ethics Committee Lazio 2, Rome, Italy (ref. number 8520).

\section{FUNDING}

No funding to declare.

\section{Hosted file}


Tables.pdf available at https://authorea.com/users/363356/articles/484164-the-relevance-ofmaternal-cardiovascular-situation-before-labor-in-the-outcome-of-birth-an-observationalstudy 